\title{
Esophageal Necrosis, CTCAE
}

National Cancer Institute

\section{Source}

National Cancer Institute. Esophageal Necrosis, CT CAE. NCI Thesaurus. Code C143455.

A disorder characterized by a necrotic process occurring in the esophageal wall. 